American J. of Engineering and Applied Sciences 1 (4): 399-407, 2008

ISSN 1941-7020

(C) 2008 Science Publications

\title{
Analytical Investigation of Hyperbolic Equations via He's Methods
}

\author{
D.D. Ganji, M. Amini and A. Kolahdooz \\ Department of Mechanical Engineering, Mazandaran University, \\ P.O. Box 484, Babol, Iran
}

\begin{abstract}
Perturbation methods depend on a small parameter which is difficult to be found for real-life nonlinear problems. To overcome this shortcoming, two new but powerful analytical methods were introduced to solve nonlinear heat transfer problems in this letter, one is He's Variational Iteration Method (VIM) and the other is the Homotopy-Perturbation Method (HPM). Nonlinear hyperbolic equations were used as examples to illustrate the simple solution procedures. These methods were useful and practical for solving the nonlinear hyperbolic equation, which is associated with variable initial condition. Comparison of the results has been obtained by both methods with exact solutions reveals that both methods were tremendously effective.
\end{abstract}

Key words: Hyperbolic equations, homotopy perturbation method, variation iteration method

\section{INTRODUCTION}

Hyperbolic partial differential equations are the subject of many researches because of their application in many engineering fields such as wave equation and telegraph equation. In recent years, several such techniques have drawn special attention, such as Hirtoa's bilinear method ${ }^{[21]}$, the Adomian's decomposition method $^{[5]}$, the EXP function method $^{[22-}$ 26], fractional method ${ }^{[27-33]}$, the Homotopy Perturbation Method (HPM $)^{[3,34-38]}$ and Variational Iteration Method $(\text { VIM })^{[39-47]}$. Various methods for obtaining explicit solutions to hyperbolic partial differential equations have been proposed ${ }^{[1-8]}$.

Biazar et al. ${ }^{[1]}$ use Adomian decomposition method to solve this equation. In this work we use homotopy perturbation method and variational iteration method to solve hyperbolic equations. Unlike classical techniques, the nonlinear equations are solved easily and elegantly without transforming or linearizing the equation by using the Homotopy Perturbation Method (HPM) ${ }^{[9-11]}$. It provides an efficient explicit solution with high accuracy, minimal calculations and avoidance of physically unrealistic assumptions.

The HPM was first proposed by $\mathrm{He}^{[12-16]}$ and has been shown to solve a large class of nonlinear problems effectively, easily and accurately with approximations converging rapidly to accurate solutions. The HPM was proposed to search for limit cycles or bifurcation curves of nonlinear equations ${ }^{[17]}$. In ${ }^{[14]}$, a heuristic example was given to illustrate the basic idea of the HPM and its advantages over the d-method, the method was also applied to solve boundary value problems ${ }^{[18]}$ and heat radiation equations ${ }^{[19]}$. Variation iteration method is based on the use of Lagrange multipliers for identification of optimal values of parameters in a functional. Using this method a rapid convergent sequence is produced. The variational iteration method is suitable for finding the approximation of the solution without discretization of the problem ${ }^{[20]}$.

The general form of hyperbolic equation as a kind of second-order quasilinear partial differential equation is:

$$
a \frac{\partial^{2} u}{\partial x^{2}}+b \frac{\partial^{2} u}{\partial x \partial y}+c \frac{\partial^{2} u}{\partial y^{2}}+e=0
$$

Where $a, b, c$ and e may be functions of $x, y, u, \frac{\partial u}{\partial x}$ and $\frac{\partial u}{\partial y}$ but not of $\frac{\partial^{2} u}{\partial x^{2}}, \frac{\partial^{2} u}{\partial x \partial y}$ and $\frac{\partial^{2} u}{\partial y^{2}}$, i.e., The second order derivatives occur only to the first degree. If $b^{2}$ $4 \mathrm{ac}>0$, then Eq. 1 is called hyperbolic equation.

\section{MATERIALS AND METHODS}

In this study, we have applied the Homotopy perturbation method and variational iteration method to the discussed problems. To illustrate the basic ideas of methods, we have considered the following nonlinear differential equation.

Corresponding Author: D.D. Ganji, Department of Mechanical Engineering, Mazandaran University, P.O. Box 484, Babol, Iran Fax: +98 1113234205 
Homotopy perturbation method: To illustrate the basic ideas of this method, we consider the following nonlinear differential equation:

$$
\mathrm{A}(\mathrm{u})-\mathrm{f}(\mathrm{r})=0, \mathrm{r} \in \Omega
$$

With the boundary conditions of:

$$
\mathrm{B}\left(\mathrm{u}, \frac{\partial \mathrm{u}}{\partial \mathrm{n}}\right)=0, \mathrm{r} \in \Gamma
$$

Where $\mathrm{A}, \mathrm{B}, \mathrm{f}(\mathrm{r})$ and $\mathrm{G}$ are a general differential operator, a boundary operator a known analytical function and the boundary of the domain $\Omega$, respectively.

Generally speaking, the operator A can be divided into a linear part L and a nonlinear part N. Eq. 2 can therefore be rewritten as:

$$
\mathrm{L}(\mathrm{u})+\mathrm{N}(\mathrm{u})-\mathrm{f}(\mathrm{r})=0
$$

By the homotopy technique, we construct a homotopy v(r,p): $\Omega \times[0,1]$ ? $\Re$ which satisfies:

$$
\begin{aligned}
& \mathrm{H}(\mathrm{v}, \mathrm{p})=(1-\mathrm{p})\left[\mathrm{L}(\mathrm{v})-\mathrm{L}\left(\mathrm{u}_{0}\right)\right]+ \\
& \mathrm{p}[\mathrm{A}(\mathrm{u})-\mathrm{f}(\mathrm{r})]=0, \mathrm{p} \in[0,1], \mathrm{r} \in \Omega
\end{aligned}
$$

or

$$
\begin{aligned}
& \mathrm{H}(\mathrm{v}, \mathrm{p})=\mathrm{L}(\mathrm{v})-\mathrm{L}\left(\mathrm{u}_{0}\right)+\mathrm{pL}\left(\mathrm{u}_{0}\right)+ \\
& \mathrm{p}[\mathrm{N}(\mathrm{v})-\mathrm{f}(\mathrm{r})]=0
\end{aligned}
$$

Where $\mathrm{p} \in[0,1]$ is an embedding parameter, while $\mathrm{u}_{0}$ is an initial approximation of Eq. 2 which satisfies the boundary conditions.

Obviously, considering Eq. 5 and 6 we will have:

$$
\begin{aligned}
& \mathrm{H}(\mathrm{v}, 0)=\mathrm{L}(\mathrm{v})-\mathrm{L}\left(\mathrm{u}_{0}\right)=0 \\
& \mathrm{H}(\mathrm{v}, 1)=\mathrm{A}(\mathrm{v})-\mathrm{f}(\mathrm{r})=0
\end{aligned}
$$

The changing process of $\mathrm{p}$ from zero to unity is just that of $v(r, p)$ from $u_{0}(r)$ to $u(r)$. In topology, this is called deformation and $\mathrm{L}(\mathrm{v})-\mathrm{L}\left(\mathrm{u}_{0}\right)$ and $\mathrm{A}(\mathrm{v})-\mathrm{f}(\mathrm{r})$ are being called homotopy.

According to the HPM, we can first use the embedding parameter $\mathrm{p}$ as a small parameter and assume that the solution of Eq. 5 and 6 can be written as a power series in $\mathrm{p}$, namely:

$$
\mathrm{v}=\mathrm{v}_{0}+\mathrm{pv}_{1}+\mathrm{p}^{2} \mathrm{v}_{2}+\cdots
$$

Setting $\mathrm{p}=1$ result in the approximate solution of Eq. 2 to:

$$
\begin{aligned}
& \mathrm{u}=\lim \mathrm{v}=\mathrm{v}_{0}+\mathrm{v}_{1}+\mathrm{v}_{2}+\cdots \\
& \mathrm{p} ? 1
\end{aligned}
$$

The combination of the perturbation method and the homotopy method is called the HPM, which eliminates the drawbacks of the traditional perturbation methods. The series 10 is convergent for most cases. However, the convergent rate depends on the nonlinear operator A (v) (the following opinions are suggested by $\left.\mathrm{He}^{[3]}\right)$ :

- The second derivative of $\mathrm{N}(\mathrm{v})$ with respect to $\mathrm{V}$ must be small because the parameter may be relatively large, i.e., $\mathrm{p} \rightarrow 1$

- The norm of $\mathrm{L}^{-1} \frac{\partial \mathrm{N}}{\partial \mathrm{v}}$ must be smaller than one so that the series converges

Variational iteration method: To clarify the basic ideas of VIM, we consider the following differential equation:

$$
\mathrm{Lu}+\mathrm{Fu}=\mathrm{g}(\mathrm{t})
$$

Where $\mathrm{L}$ is a linear operator, $\mathrm{F}$ is a nonlinear operator and $\mathrm{g}(\mathrm{t})$ is a heterogeneous term.

According to VIM, we can write down a correction functional as follows:

$$
\mathrm{u}_{\mathrm{n}+1}(\mathrm{t})=\mathrm{u}_{\mathrm{n}}(\mathrm{t})+\int_{0}^{\mathrm{t}} \lambda\left(\operatorname{Lu}_{\mathrm{n}}(\tau)+F \tilde{u}_{\mathrm{n}}(\tau)-\mathrm{g}(\tau)\right) \mathrm{d} \tau
$$

Where $\lambda$ is a general Lagrangian multiplier ${ }^{[17-20]}$ which can be identified optimally via the variational theory.

The subscript $\mathrm{n}$ indicates the $n$th approximation and $\tilde{u}_{\mathrm{n}}$ is considered as a restricted variation ${ }^{[17-20]}$, i.e., $\delta \tilde{u}_{\mathrm{n}}=0$.

Applications: In order to assess the accuracy of VIM and HPM for solving hyperbolic equations and to compare it with exact solution, we will consider the three following examples.

Example1: Consider the partial differential equation with the initial conditions ${ }^{[7]}$ :

$$
\frac{\partial^{2} u}{\partial x^{2}}+(1-2 x) \frac{\partial^{2} u}{\partial x \partial y}+\left(x^{2}-x-2\right) \frac{\partial^{2} u}{\partial y^{2}}=0
$$


Am. J. Engg. \& Applied Sci., 1 (4): 399-407, 2008

$u(x, 0)=x$,
$\frac{\partial u(x, 0)}{\partial y}=1$

Homotopy perturbation method: Now we apply homotopy perturbation to Eq. 13:

$$
\begin{aligned}
& H(v, p):=(1-p)\left(x^{2}-x-2\right)\left[\frac{\partial^{2}}{\partial y^{2}} v(x, y)\right] \\
& +p\left[\begin{array}{l}
\frac{\partial^{2}}{\partial x^{2}} v(x, y)+(1-2 x)\left[\frac{\partial^{2}}{\partial y \partial x} v(x, y)\right] \\
+\left(x^{2}-x-2\right)\left[\frac{\partial^{2}}{\partial y^{2}} v(x, y)\right]
\end{array}\right]=0
\end{aligned}
$$

Substituting Eq. 9 into Eq. 14 and rearranging based on powers of $p$-terms, we have:

$$
\begin{gathered}
p^{0}: x^{2}\left[\frac{\partial^{2}}{\partial y^{2}} v_{0}(x, y)\right]-x\left[\frac{\partial^{2}}{\partial y^{2}}\right. \\
\left.v_{0}(x, y)\right]-2\left[\frac{\partial^{2}}{\partial y^{2}} v_{y}(x, y)\right]=0 \\
\frac{\partial^{2}}{\partial x^{2}} v_{0}(x, y)-2\left[\frac{\partial^{2}}{\partial y^{2}} v_{1}(x, y)\right] \\
+x^{2}\left[\frac{\partial^{2}}{\partial y^{2}} v_{1}(x, y)\right] \\
+\frac{\partial^{2}}{\partial y \partial x} v_{0}(x, y)-2 x\left[\frac{\partial^{2}}{\partial y \partial x} v_{0}(x, y)\right] \\
-x\left[\frac{\partial^{2}}{\partial y^{2}} v(x, y)\right]=0 \\
\left.p^{3}: \quad+\frac{\partial^{2}}{\partial y \partial x} v_{1}(x, y)\right]+x^{2}\left[\frac{\partial^{2}}{\partial y^{2}} v_{2}(x, y)\right] \\
+\frac{\partial^{2}}{\partial x^{2}} v_{1}(x, y)-2\left[\frac{\partial^{2}}{\partial y^{2}} v_{2}(x, y)\right] \\
+\frac{\partial^{2}}{\partial y \partial x} v_{1}(x, y)-x\left[\frac{\partial^{2}}{\partial y^{2}} v_{2}(x, y)\right]=0 \\
\frac{\partial^{2}}{\partial x^{2}} v_{2}(x, y)-2\left[\frac{\partial^{2}}{\partial y \partial x} v_{3}(x, y)-2\left[\frac{\partial^{2}}{\partial y^{2}} v_{3}(x, y)\right]\right. \\
\left.+\frac{\partial^{2}}{\partial y^{2}} v_{3}(x, y)\right]=0
\end{gathered}
$$

To determine $u$, the above equations should be solved. Considering the appropriate initial conditions we have:

$$
\begin{aligned}
& \mathrm{v}_{0}(\mathrm{x}, 0)=\mathrm{x}+\mathrm{y}, \frac{\partial}{\partial y} \mathrm{v}_{0}(\mathrm{x}, 0)=1 \\
& \mathrm{v}_{\mathrm{i}}(\mathrm{x}, 0)=0,\left.\frac{\partial}{\partial y} \mathrm{v}_{\mathrm{i}}(\mathrm{x}, 0)\right|_{\mathrm{y}=0}=0, \mathrm{i}=1,2, \ldots
\end{aligned}
$$

The Solution of Eq. 15-19 may be written as follows:

$$
\mathrm{v}_{0}(\mathrm{x}, 0)=\mathrm{x}+\mathrm{y}
$$

In the same manner, the rest of components were obtained using the maple package.

According to the HPM, we can conclude:

$$
\begin{aligned}
& \mathrm{u}(\mathrm{x}, \mathrm{y})=\operatorname{limv}_{\mathrm{p} \rightarrow 1}(\mathrm{x}, \mathrm{y})=\mathrm{v}_{0}(\mathrm{x}, \mathrm{y})+ \\
& \mathrm{v}_{1}(\mathrm{x}, \mathrm{y})+\mathrm{v}_{\mathrm{l}}(\mathrm{x}, \mathrm{y})+\ldots
\end{aligned}
$$

In this manner three components of the perturbation series 9 were obtained. So, we have:

$$
u(x, y)=x+y
$$

Variational iteration method: Now Before applying this procedure to Eq. 13 following ${ }^{[2,43]}$, we construct a correction functional, as follows:

$$
\begin{aligned}
& u_{n+1}(x, y)=u_{n}(x, y)+\int_{0}^{y} \lambda(\tau)\left[\frac{\partial^{2}}{\partial x^{2}} u_{n}(x, \tau)+(1-2 x)\right. \\
& \left.\left[\frac{\partial^{2}}{\partial x \partial \tau} u_{n}(x, \tau)\right]+\left(x^{2}-x-2\right)\left[\frac{\partial^{2}}{\partial \tau^{2}} u_{n}(x, \tau)\right]\right] d \tau
\end{aligned}
$$
follows:

Its stationary conditions can be obtained as

$$
\begin{aligned}
& 1-\left(x^{2}-x-2\right)\left[\frac{d}{d y} \lambda(y)\right]=0, \\
& \left(x^{2}-x-2\right)\left[\frac{d^{2}}{d \tau^{2}} \lambda(\tau)\right]=0, \\
& \lambda(y)\left[\frac{\partial^{2}}{\partial x^{2}} u_{n}(x, y)\right]=0, \lambda(y)=0
\end{aligned}
$$

The Lagrangian multiplier can therefore be identified as:

$$
\lambda(\tau)=\frac{\tau-\mathrm{y}}{\mathrm{x}^{2}-\mathrm{x}-2}
$$

As a result, we obtain the following iteration formula: 
Am. J. Engg. \& Applied Sci., 1 (4): 399-407, 2008

$$
\begin{aligned}
& u_{n+1}(x, y)=u_{n}(x, y)+\int_{0}^{y} \lambda(\tau)\left[\frac{\partial^{2}}{\partial x^{2}} u_{n}(x, \tau)+(1-2 x)\right. \\
& \left.\left[\frac{\partial^{2}}{\partial x \partial \tau} u_{n}(x, \tau)\right]+\left(x^{2}-x-2\right)\left[\frac{\partial^{2}}{\partial \tau^{2}} u_{n}(x, \tau)\right]\right] d \tau
\end{aligned}
$$

Now we start with an arbitrary initial approximation that satisfies the initial condition:

$$
\mathrm{u}_{0}(\mathrm{x}, \mathrm{y})=\mathrm{x}+\mathrm{y}
$$

Using the above variational formula 25, we have:

$$
\begin{aligned}
& \mathrm{u}_{1}(\mathrm{x}, \mathrm{y})=\mathrm{u}_{0}(\mathrm{x}, \mathrm{y})+\int_{0}^{\mathrm{y}} \lambda(\tau)\left[\frac{\partial^{2}}{\partial \mathrm{x}^{2}} \mathrm{u}_{0}(\mathrm{x}, \tau)+(1-2 \mathrm{x})\right. \\
& \left.\left[\frac{\partial^{2}}{\partial \mathrm{x} \partial \tau} \mathrm{u}_{0}(\mathrm{x}, \tau)\right]+\left(\mathrm{x}^{2}-\mathrm{x}-2\right)\left[\frac{\partial^{2}}{\partial \tau^{2}} \mathrm{u}_{0}(\mathrm{x}, \tau)\right]\right] \mathrm{d} \tau
\end{aligned}
$$

Substituting Eq. 26 in to Eq. 27 and after simplifications, we have:

$$
\mathrm{u}_{\mathrm{l}}(\mathrm{x}, \mathrm{y})=\mathrm{x}+\mathrm{y}
$$

In the same way, we can obtain $\mathrm{U}_{2}(\mathrm{X}, \mathrm{Y}), \mathrm{U}_{3}(\mathrm{X}, \mathrm{Y})$ and the rest of the components of the iteration formula.

Example 2: Consider the partial differential equation with the initial conditions ${ }^{[7]}$ :

$$
\begin{aligned}
& \frac{\partial^{2} u}{\partial x^{2}}+\frac{\partial^{2} u}{\partial x \partial y}-2 \frac{\partial^{2} u}{\partial y^{2}}+1=0, \\
& u(x, 0)=x, \\
& \frac{\partial u(x, 0)}{\partial y}=x .
\end{aligned}
$$

Homotopy perturbation method: By applying homotopy perturbation to Eq. 29. We have:

$$
\begin{aligned}
& H(v, p)=-2(1-p)\left[\frac{\partial^{2}}{\partial y^{2}} v(x, y)\right]+p\left[\frac{\partial^{2}}{\partial x^{2}} v(x, y)\right. \\
& \left.+\frac{\partial^{2}}{\partial y \partial x} v(x, y)-2\left[\frac{\partial^{2}}{\partial y^{2}} v(x, y)\right]+1\right]=0
\end{aligned}
$$

Substituting Eq. 9 into Eq. 29 and rearranging based on powers of $\mathrm{p}$-terms, we have:

$$
\begin{aligned}
& p^{0}:-2\left[\frac{\partial^{2}}{\partial y^{2}} v_{0}(x, y)\right]=0 \\
& p^{1}:-2\left[\frac{\partial^{2}}{\partial y^{2}} v_{1}(x, y)\right]+\frac{\partial^{2}}{\partial x^{2}} \\
& v_{0}(x, y)+\frac{\partial^{2}}{\partial y \partial x} v_{0}(x, y)+1=0
\end{aligned}
$$

$$
\begin{aligned}
& \mathrm{p}^{2}:-2\left[\frac{\partial^{2}}{\partial \mathrm{y}^{2}} v_{2}(\mathrm{x}, \mathrm{y})\right]+\frac{\partial^{2}}{\partial \mathrm{x}^{2}} \\
& v_{1}(\mathrm{x}, \mathrm{y})+\frac{\partial^{2}}{\partial \mathrm{y} \partial \mathrm{x}} v_{1}(\mathrm{x}, \mathrm{y})=0
\end{aligned}
$$

$$
\begin{aligned}
& p^{3}:-2\left[\frac{\partial^{2}}{\partial y^{2}} v_{3}(x, y)\right]+\frac{\partial^{2}}{\partial y^{2}} \\
& v_{2}(x, y)+\frac{\partial^{2}}{\partial y \partial x} v_{2}(x, y)=0
\end{aligned}
$$

To determine $u$, the above equations should be solved. Considering the appropriate initial conditions we have:

$$
\begin{aligned}
& v_{0}(x, 0)=x+x y, \frac{\partial}{\partial y} v_{0}(x, 0)=x, \\
& v_{i}(x, 0)=0,\left.\frac{\partial}{\partial y} v_{i}(x, 0)\right|_{y=0}=0, i=1,2, \ldots
\end{aligned}
$$

The solution of Eq. 31-35 may be written as follows:

$$
\begin{aligned}
& \mathrm{n}_{0}(\mathrm{x}, \mathrm{y})=\mathrm{x}+\mathrm{xy} \\
& \mathrm{n}_{1}(\mathrm{x}, \mathrm{y})=\frac{1}{2} \mathrm{y}^{2} \ldots \text { and soon }
\end{aligned}
$$

In the same manner, the rest of components was obtained using the maple package. According to the HPM, we can conclude:

$$
\begin{aligned}
& \mathrm{u}(\mathrm{x}, \mathrm{y})=\operatorname{limv}_{\mathrm{p} \rightarrow 1}(\mathrm{x}, \mathrm{y})=\mathrm{v}_{\mathrm{d}}(\mathrm{x}, \mathrm{y}) \\
& +\mathrm{v}_{\mathrm{f}}(\mathrm{x}, \mathrm{y})+\mathrm{v}_{\mathrm{d}}(\mathrm{x}, \mathrm{y})+\cdots
\end{aligned}
$$

Therefore the solution is:

$$
\mathrm{u}(\mathrm{x}, \mathrm{y}):=\mathrm{x}+\mathrm{xy}+\frac{1}{2} \mathrm{y}^{2}
$$

Variational iteration method: First we construct a correction functional like example 1, as follows:

$$
\begin{aligned}
& \mathrm{u}_{\mathrm{n}+1}(\mathrm{x}, \mathrm{y})=\mathrm{u}_{\mathrm{n}}(\mathrm{x}, \mathrm{y})+\int_{0}^{\mathrm{y}} \lambda(\tau)\left[\frac{\partial^{2}}{\partial \mathrm{x}^{2}} \mathrm{u}_{\mathrm{n}}(\mathrm{x}, \tau)+\right. \\
& \left.\frac{\partial^{2}}{\partial \mathrm{x} \partial \tau} \mathrm{u}_{\mathrm{n}}(\mathrm{x}, \tau)-2\left(\frac{\partial^{2}}{\partial \tau^{2}} \mathrm{u}_{\mathrm{n}}(\mathrm{x}, \tau)\right)+1\right] \mathrm{d} \tau
\end{aligned}
$$
follows:

Its stationary conditions can be obtained as 
Am. J. Engg. \& Applied Sci., 1 (4): 399-407, 2008

$$
\begin{aligned}
& 1+2\left(\frac{d}{d y} \lambda(y)\right)=0,-2\left(\frac{d^{2}}{d \tau^{2}} \lambda(\tau)\right)=0, \\
& \lambda(y)\left[\frac{\partial^{2}}{\partial x^{2}} u_{n}(x, y)\right]=0, \lambda(y)=0
\end{aligned}
$$

The Lagrangian multiplier can therefore be identified as:

$$
\lambda(\tau)=\frac{\tau-\mathrm{y}}{-2}
$$

As a result, we obtain the following iteration formula:

$$
\begin{aligned}
& \mathrm{u}_{\mathrm{n}+1}(\mathrm{x}, \mathrm{y})=\mathrm{u}_{\mathrm{n}}(\mathrm{x}, \mathrm{y})+\int_{0}^{\mathrm{y}} \lambda(\tau)\left[\frac{\partial^{2}}{\partial \mathrm{x}^{2}} \mathrm{u}_{\mathrm{n}}(\mathrm{x}, \tau)+\right. \\
& \left.\frac{\partial^{2}}{\partial \mathrm{x} \partial \tau} \mathrm{u}_{\mathrm{n}}(\mathrm{x}, \tau)-2\left(\frac{\partial^{2}}{\partial \tau^{2}} \mathrm{u}_{\mathrm{n}}(\mathrm{x}, \tau)\right)+1\right] \mathrm{d} \tau
\end{aligned}
$$

Now we start with an arbitrary initial approximation that satisfies the initial condition:

$$
\mathrm{u}_{0}(\mathrm{x}, \mathrm{y})=\mathrm{xy}+\mathrm{x}
$$

Using the above variational formula 43 , we have

$$
\begin{aligned}
& \mathrm{u}_{1}(\mathrm{x}, \mathrm{y})=\mathrm{u}_{0}(\mathrm{x}, \mathrm{y})+\int_{0}^{\mathrm{y}} \lambda(\tau)\left[\frac{\partial^{2}}{\partial \mathrm{x}^{2}} \mathrm{u}_{0}(\mathrm{x}, \tau)+\right. \\
& \left.\frac{\partial^{2}}{\partial \mathrm{x} \partial \tau} \mathrm{u}_{0}(\mathrm{x}, \tau)-2\left(\frac{\partial^{2}}{\partial \tau^{2}} \mathrm{u}_{0}(\mathrm{x}, \tau)\right)+1\right] \mathrm{d} \tau
\end{aligned}
$$

Substituting Eq. 44 in to Eq. 45 and after simplifications, we have:

$$
u_{1}(x, y)=x y+x+\frac{1}{4} y^{2}
$$
follows:

In the same way, we can obtain $\mathrm{U}_{2}(\mathrm{X}, \mathrm{Y})$ as

$$
\mathrm{u}_{2}(\mathrm{x}, \mathrm{y})=\mathrm{xy}+\mathrm{x}+\frac{1}{2} \mathrm{y}^{2}
$$

And so on. In the same manner the rest of the components of the iteration formula can be obtained.

Example 3: Consider the partial differential equation with the initial conditions ${ }^{[6]}$ :

$$
\begin{aligned}
& \frac{\partial^{2} u}{\partial x^{2}}-4 x^{2} \frac{\partial^{2} u}{\partial y^{2}}=0, \\
& u(x, 0)=x^{2} \\
& \frac{\partial u(x, 0)}{\partial y}=0
\end{aligned}
$$

Homotopy perturbation method: By applying homotopy perturbation to Eq. 48, we have:

$$
\begin{aligned}
& H(v, p)=-4(1-p) x^{2}\left[\frac{\partial^{2}}{\partial y^{2}} v(x, y)\right] \\
& +p\left[\frac{\partial^{2}}{\partial x^{2}} v(x, y)-4 x^{2}\left[\frac{\partial^{2}}{\partial y^{2}} v(x, y)\right]\right]=0
\end{aligned}
$$

Substituting Eq. 9 into Eq. 48 and rearranging based on powers of $\mathrm{p}$-terms, we have:

$$
\begin{aligned}
& p^{0}:-4 x^{2}\left[\frac{\partial^{2}}{\partial y^{2}} v_{0}(x, y)\right]=0 \\
& p^{1}:-4 X^{2}\left[\frac{\partial^{2}}{\partial y^{2}} v_{1}(x, y)\right]+\frac{\partial^{2}}{\partial x^{2}} v_{0}(x, y)=0 \\
& p^{2}:-4 x^{2}\left[\frac{\partial^{2}}{\partial y^{2}} v_{2}(x, y)\right]+\frac{\partial^{2}}{\partial x^{2}} v_{1}(x, y)=0 \\
& p^{3}:-4 x^{2}\left[\frac{\partial^{2}}{\partial y^{2}} v_{3}(x, y)\right]+\frac{\partial^{2}}{\partial x^{2}} v_{2}(x, y)=0 \\
& p^{4}:-4 x^{2}\left[\frac{\partial^{2}}{\partial y^{2}} v_{4}(x, y)\right]+\frac{\partial^{2}}{\partial x^{2}} v_{3}(x, y)=0
\end{aligned}
$$

To determine $u$, the above equations should be solved. Considering the appropriate initial conditions we have:

$$
\begin{aligned}
& \mathrm{v}_{0}(\mathrm{x}, 0)=\mathrm{x}^{2}, \frac{\partial}{\partial \mathrm{y}} \mathrm{v}_{\mathrm{d}}(\mathrm{x}, 0)=0, \\
& \mathrm{v}_{\mathrm{i}}(\mathrm{x}, 0)=0,\left.\frac{\partial}{\partial \mathrm{y}} \mathrm{v}_{\mathrm{i}}(\mathrm{x}, 0)\right|_{\mathrm{y}=0}=0, \mathrm{i}=1,2, \ldots
\end{aligned}
$$

The Solution of Eq. 50-55 may be written as follows:

$v_{0}(x, y)=x^{2}$

$$
v_{1}(x, y)=\frac{1}{4} \frac{y^{2}}{x^{2}}
$$

$v_{2}(x, y)=\frac{1}{32} \frac{y^{4}}{x^{6}}$

$$
v_{3}(x, y)=\frac{7}{640} \frac{y^{6}}{x^{10}}
$$

$$
v_{4}(x, y)=\frac{11}{2048} \frac{y^{8}}{x^{14}}
$$


Therefore the solution is:

$$
u(x, y)=x^{2}+\frac{1}{4} \frac{y^{2}}{x^{2}}+\frac{1}{32} \frac{y^{4}}{x^{6}}+\frac{7}{640} \frac{y^{6}}{x^{10}}+\frac{11}{2048 x^{14}}
$$

Variational iteration method: First we construct a correction functional like example 1, as follows:

$$
\begin{aligned}
& u_{n+1}(x, y)=u_{n}(x, y)+\int_{0}^{y} \lambda(\tau)\left[\frac{\partial^{2}}{\partial x^{2}}\right. \\
& \left.u_{n}(x, \tau)-4 x^{2}\left(\frac{\partial^{2}}{\partial \tau^{2}} u_{n}(x, \tau)\right)\right] d \tau
\end{aligned}
$$
follows:

Its stationary conditions can be obtained as

$$
\begin{aligned}
& 1+4 x^{2}\left(\frac{d}{d y} \lambda(y)\right)=0,-4 x^{2}\left(\frac{d^{2}}{d \tau^{2}} \lambda(\tau)\right)=0 \\
& \lambda(y)\left[\frac{\partial^{2}}{\partial x^{2}} u_{n}(x, y)\right]=0, \lambda(y)=0
\end{aligned}
$$

The Lagrangian multiplier can therefore be identified as:

$$
\lambda(\tau)=-\frac{1}{4} \frac{\tau}{\mathrm{x}^{2}}+\frac{1}{4} \frac{\mathrm{y}}{\mathrm{x}^{2}}
$$

As a result, we obtain the following iteration formula:

$$
\begin{aligned}
& u_{n+1}(x, y)=u_{n}(x, y)+\int_{0}^{y} \lambda(\tau)\left[\frac{\partial^{2}}{\partial x^{2}}\right. \\
& \left.u_{n}(x, \tau)-4 x^{2}\left(\frac{\partial^{2}}{\partial \tau^{2}} u_{n}(x, \tau)\right)\right] d \tau
\end{aligned}
$$

Now we start with an arbitrary initial approximation that satisfies the initial condition:

$$
\mathrm{u}_{0}(\mathrm{x}, \mathrm{y})=\mathrm{x}^{2}
$$

Using the above variational formula 65 , we have:

$$
\begin{aligned}
& \mathrm{u}_{1}(\mathrm{x}, \mathrm{y})=\mathrm{u}_{0}(\mathrm{x}, \mathrm{y})+\int_{0}^{\mathrm{y}} \lambda(\tau)\left[\frac{\partial^{2}}{\partial \mathrm{x}^{2}}\right. \\
& \left.\mathrm{u}_{0}(\mathrm{x}, \tau)-4 \mathrm{x}^{2}\left(\frac{\partial^{2}}{\partial \tau^{2}} \mathrm{u}_{0}(\mathrm{x}, \tau)\right)\right] \mathrm{d} \tau
\end{aligned}
$$

Substituting Eq. 66 in to Eq. 67 and after simplifications, we have:

$$
\mathrm{u}(\mathrm{x}, \mathrm{y})=\frac{1}{4} \frac{4 \mathrm{x}^{2}+\mathrm{y}^{2}}{\mathrm{x}^{2}}
$$

In the same way, we can obtain $\mathrm{U}_{2}(\mathrm{X}, \mathrm{Y}), \mathrm{U}_{3}(\mathrm{X}, \mathrm{Y})$ and $\mathrm{U}_{4}(\mathrm{X}, \mathrm{Y})$ as follows:

$$
u_{2}(x, y)=\frac{1}{16} \frac{16 x^{8}+8 x^{4} y^{2}+3 y^{4}}{x^{6}}
$$

$$
\begin{aligned}
& u_{3}(x, y)=\frac{1}{64} \frac{64 x^{12}+48 x^{8} y^{2}+36 x^{4} y^{4}+63 y^{4}}{x^{10}} \\
& u_{3}(x, y)=x^{2}+\frac{1}{4} \frac{y^{2}}{x^{2}}+\frac{1}{32} \frac{y^{4}}{x^{6}}+\frac{7}{640} \frac{y^{6}}{x^{10}}+\frac{11}{2048} \frac{y^{8}}{x^{14}}
\end{aligned}
$$

And so on. In the same manner the rest of the components of the iteration formula can be obtained.

\section{RESULTS}

The solution of hyperbolic equations has been investigated analytically by Homotopy Perturbation Method (HPM) and Variational Iteration Method (VIM). U(X, Y) for both Eq. 47 and 71 have been shown in Fig. 1 and 2 respectively. Also in Tables 1-3, for some values of $\mathrm{x}$ and $\mathrm{y}$, results for both methods have been compared with exact solution. The results have shown that they are considerably capable of solving a wide range of hyperbolic equations.

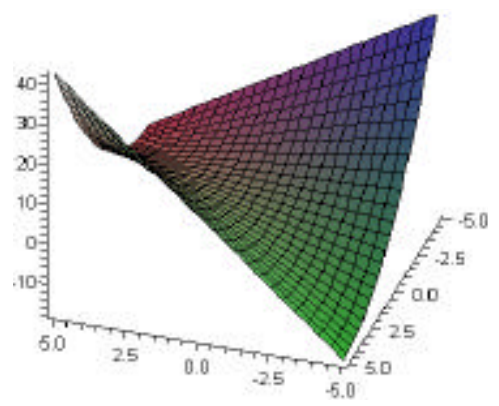

Fig. 1: 3D plot of u (x, y) for Eq. 47

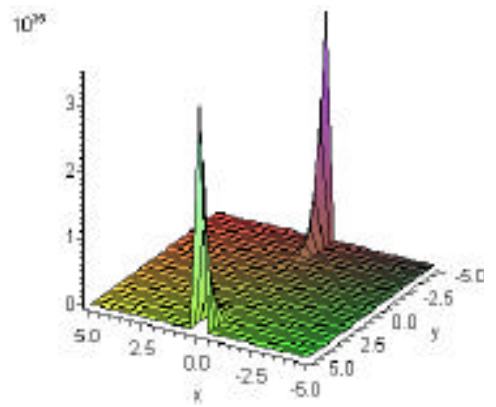

Fig. 2: 3D plot of $\mathrm{u}(\mathrm{x}, \mathrm{y})$ for Eq. 71 
Am. J. Engg. \& Applied Sci., 1 (4): 399-407, 2008

Table 1: The solution of $\mathrm{u}(\mathrm{x}, \mathrm{y})$ for different values of $\mathrm{x}$ and $\mathrm{y}$

\begin{tabular}{lllll}
\hline $\mathrm{x}$ & $\mathrm{y}$ & $\begin{array}{l}\mathrm{U}(\mathrm{x}, \mathrm{y}) \\
(\mathrm{HPM})\end{array}$ & $\begin{array}{l}\mathrm{U}(\mathrm{x}, \mathrm{y}) \\
(\mathrm{VIM})\end{array}$ & $\begin{array}{l}\mathrm{U}(\mathrm{x}, \mathrm{y}) \\
\text { (exact result) }\end{array}$ \\
\hline 0.035 & 0.071 & 0.106 & 0.106 & 0.107 \\
0.139 & 0.074 & 0.213 & 0.213 & 0.212 \\
0.448 & 0.077 & 0.525 & 0.525 & 0.526 \\
0.758 & 0.075 & 0.833 & 0.833 & 0.834 \\
0.819 & 0.152 & 0.971 & 0.971 & 0.971 \\
\hline
\end{tabular}

Table 2: The solution of $\mathrm{u}(\mathrm{x}, \mathrm{y})$ for different values of $\mathrm{x}$ and $\mathrm{y}$

\begin{tabular}{lllll}
\hline $\mathrm{x}$ & $\mathrm{y}$ & $\begin{array}{l}\mathrm{U}(\mathrm{x}, \mathrm{y}) \\
(\mathrm{HPM})\end{array}$ & $\begin{array}{l}\mathrm{U}(\mathrm{x}, \mathrm{y}) \\
(\mathrm{VIM})\end{array}$ & $\begin{array}{l}\mathrm{U}(\mathrm{x}, \mathrm{y}) \\
\text { (exact result) }\end{array}$ \\
\hline 0.033 & 0.067 & 0.0374 & 0.0374 & 0.0378 \\
0.133 & 0.067 & 0.1442 & 0.1442 & 0.1444 \\
0.833 & 0.067 & 0.8911 & 0.8911 & 0.8911 \\
0.067 & 0.133 & 0.0848 & 0.0848 & 0.0844 \\
0.767 & 0.133 & 0.8779 & 0.8779 & 0.8778 \\
\hline
\end{tabular}

Table 3: The solution of $u(x, y)$ for different values of $x$ and $y$

\begin{tabular}{lllll}
\hline $\mathrm{x}$ & $\mathrm{y}$ & $\begin{array}{l}\mathrm{U}(\mathrm{x}, \mathrm{y}) \\
(\mathrm{HPM})\end{array}$ & $\begin{array}{l}\mathrm{U}(\mathrm{x}, \mathrm{y}) \\
(\mathrm{VIM})\end{array}$ & $\begin{array}{l}\mathrm{U}(\mathrm{x}, \mathrm{y}) \\
\text { (exact result) }\end{array}$ \\
\hline 0.35714 & 0.034286 & 0.12987 & 0.12987 & 0.12918 \\
0.35357 & 0.035180 & 0.12749 & 0.12749 & 0.12709 \\
\hline
\end{tabular}

\section{DISCUSSION}

In this study, the authors have intended to show that the two methods, HPM and VIM, are considerably capable of solving a wide range of hyperbolic equations. The examples given in this study reveal that both methods are very effective and have high accuracy. In some cases as Examples 1 and 2, after some steps the remaining terms would vanish and we derive the exact solution. In the cases as Example 3, the approximation can be obtained to any desired number of terms.

VIM and HPM do not need small parameters, the limitations and non-physical assumptions required in classical perturbation methods are eliminated, furthermore, VIM and HPM can overcome the difficulties arising in the calculation of Adomian polynomials. They do not require linearization; both methods are very promising tools for hyperbolic equations. Therefore, both methods will find applications in various fields.

\section{CONCLUSION}

The methods of HPM and VIM have been successfully performed for hyperbolic equations. In these cases, we obtained excellent performances that might lead to promising approaches for many applications. All the examples showed that the results of the present methods were in excellent agreement with exact solutions. It is capable to converge to correct results with fewest number of iterations or even once, for some cases.

\section{REFERENCES}

1 Biazar, J. and H. Ebrahimi, 2005. An approximation to the solution of hyperbolic equations by adomian decomposition method and comparison with characteristics method. Appl. Math. Comput., 163: 633-638. DOI: 10.1016/j.amc.2004.04.005

2. Biazar, J., E. Babolian, and R. Islam, 2003. Solution of a system of volterra integral equations of the first kind by Adomian method. Appl. Math. Comput., 139: 249-258. DOI: 10.1016/S00963003(02)00173-X

3. He, J.H., 1999. Homotopy perturbation technique. Comput. Meth. Appl. Mech. Eng., 178: 257-262. DOI: 10.1016/S0045-7825(99)00018-3

4. Adomian, G., 1989. Nonlinear Stochastic Systems Theory and Applications to Physics. Kluwer Academic Publishers, Dordrecht, pp: 224. ISBN: 902772525X.

5. Adomian, G., 1994. Solving Frontier Problems of Physics: The Decomposition Method. Kluwer Academic Publishers, Dordrecht, pp: 372. ISBN10: 079232644X

6. Evans, G., J. Blackledge and P. Yardley, 2000. Numerical Methods for Partial Differential Equations. Springer-Verlag, Berlin, pp: 259.

7. Smith, G.D., 1978. Numerical Solution of Partial Differential Equations; Finite Difference Methods. 2nd Edn., Oxford University Press, Oxford, ISBN10: 0198596251.

8. Wazwaz, A.M., 2000. A new algorithm for calculation Adomian polynomials for nonlinear operators. Appl. Math. Comput., 111: 33-51. DOI: 10.1016/S0096-3003(99)00063-6

9. Ganji, D.D., 2006. The application of He's homotopy perturbation method to nonlinear equations arising in heat transfer. Phy. Lett., A, 355: 337-341. DOI: 10.1016/j.physleta.2006.02.056

10. Ganji, D.D. and M. Rafei, 2006. Solitary wave solutions for a generalized hirota-satsuma coupled $\mathrm{KdV}$ equation by homotopy perturbation method. Phys. Lett., A 356:131-137. DOI: 10.1016/j. physleta.2006.03.039

11. Rafei, M., D.D. Ganji, 2006. Explicit solutions of Helmholtz equation and fifth-order $\mathrm{KdV}$ equation using homotopy perturbation method. Int. J. Nonlinear Sci. Numer. Simulat., 7: 321. http://direct.bl.uk/bld/PlaceOrder.do?UIN=190647 700\&ETOC $=$ RN\&from $=$ searchengine

12. He, J.H., 2004. The homotopy perturbation method for nonlinear oscillators with discontinuities. Applied Math. Comput., 151: 287-292. DOI: 10.1016/S0096-3003(03)00341-2. 
13. He, J.H., 2004. Comparison of homotopy perturbation method and homotopy analysis method. Applied Math. Comput., 156: 527-539. DOI: 10.1016/j.amc.2003.08.008

14. He, J.H., 2004. Asymptotology by homotopy perturbation method. Applied Math. Comput., 156: 591-596. DOI: 10.1016/j.amc.2003.08.011

15. He, J.H., 2003. A simple perturbation approach to Blasius equation. Applied Math. Com., 140: 217-222. PII: S0096-3003(02)00189- 3

16. He, J.H., 2005. Application of homotopy perturbation method to nonlinear wave equations. Chaos Solutions Fractals, 26: 695-700. DOI: 10.1016/j. chaos.2005.03.006

17. He, J.H., 2005. Limit cycle and bifurcation of nonlinear problems. Chaos Solitons Fractals, 26: 827-833. DOI:10.1016/j.chaos.2005.03.007

18. He, J.H., 2006. Homotopy perturbation method for solving boundary value problems. Phys. Lett., A., 350: 87-88. DOI: 10.1016/j.physleta.2005.10.005

19. Ganji, D.D. and A. Rajabi, 2006. Assessment of homotopy-perturbation and perturbation methods in heat radiation equations. Int. Commun. Heat Mass Transfer, 33: 391-400. DOI: 10.1016/j. icheatmasstransfer.2005.11.001

20. He, J.H., 2003. Homotopy perturbation method: a new nonlinear analytical technique. Int. J. NonLinear Sci. Numer. Simul., 135: 73-79. http://cat.inist.fr/?aModele $=$ afficheN\&cpsidt $=1438$ 0691

21. $\mathrm{Hu}, \mathrm{X}$.B. and Y.T. Wu, 1998. Application of the Hirota bilinear formalism to a new integrable differential-difference equation. Phys. Lett., A., 246: 523. DOI: 10.1016/S0375-9601(98)00571-4

22. He, J.H. and M.A. Abdou, 2007. New periodic solutions for nonlinear evolution equations using Exp-function method. Chaos, Solutions and Fractals, 34: 1421-1429. DOI: 10.1016/j. chaos.2006.05.072

23. Xu-Hong (Benn) Wu and J.H. He, 2007. Solitary solutions, periodic solutions and compacton-like solutions using the Exp-function method. Comput. Math. Applied, 54: 966-986. DOI: 10.1016/j. camwa.2006.12.041

24. He, J.H. and Li-Na Zhang, 2007. Generalized solitary solution and compacton-like solution of the Jaulent-Miodek equations using the Exp-function method. Phys. Lett. A., 372: 1044-1047. DOI: 10.1016/j.physleta.2007.08.059.

25. Xu-Hong, (Benn)Wu and Ji-Huan He, 2007. EXPfunction method and its application to nonlinear equations. Chaos, Solutions and Fractals, 38: 903-910. DOI: 10.1016/j.chaos.2007.01.024
26. He, J.H. and $\mathrm{Xu}-$ Hong $\mathrm{Wu}, 2006$. Exp-function method for nonlinear wave equations. Chaos, Solitons and Fractals, 30: 700-708. DOI: 10.1016/j. chaos.2006.03.020

27. Momani, S., 2007. An algorithm for solving the fractional convection-diffusion equation with nonlinear source term. Commun. Nonlinear Sci. Numerical Simulat., 12: 1283-1290. DOI: 10. 1016/j.cnsns.2005.12.007

28. Momani, S. and Z. Odibat, 2007. Comparison between the homotopy perturbation method and the variational iteration method for linear fractional partial differential equations. Comput. Math. Appleid, 54: 910-919. DOI: 10.1016/j. camwa.2006. 12.037.

29. Momani, S. and Z. Odibat, 2007. Numerical approach to differential equations of fractional order. J. Comput. Appl. Math., 207: 96-110. DOI: 10.1016/j.cam.2006.07.015.

30. Ibrahim, R.W. and S. Momani, 2007. On the existence and uniqueness of solutions of a class of fractional differential equations. J. Math. Anal. Applied, 334: 1-10. DOI: 10.1016/j.jmaa.2006.12.036

31. Momani, S. and Z. Odibat, 2007. Numerical comparison of methods for solving linear differential equations of fractional order. $J$. Comput. Applied Math., 31: 1248-1255. DOI: 10.1016/j.chaos.2005.10.068

32. Momani, S. and R. Qaralleh, 2007. Numerical approximations and Padé approximants for a fractional population growth model. Applied Math. Model., 31: 1907-1914. DOI: 10.1016/j.apm. 2006.06.015

33. Jaradat, O.K., A.Al-Omari and S. Momani, 2008. Existence of the mild solution for fractional semilinear initial value problems. Nonlinear Anal. Theory, Methods Applied, 69: 3153-3159. DOI: 10. 1016/j.na.2007.09.008.

34. Ganji, D.D. and A. Sadighi, 2007. Application of homotopy-perturbation and variational iteration methods to nonlinear heat transfer and porous media equations. J. Comput. Applied Math., 207: 24-34. DOI: 10.1016/j.cam.2006.07.030

35. Ganji, D.D., 2006. The application of He's homotopy perturbation method to nonlinear equations arising in heat transfer. Phys. Lett., A., 355: 337-341. DOI: 10.1016/j.physleta.2006.02.056

36. Ganji, D.D., M. Jannatabadi, E. Mohseni, 2007. Application of He's variational iteration method to nonlinear Jaulent-Miodek equations and comparing it with ADM. J. Comput. Applied Math., 207: 35-45. DOI: 10.1016/j.cam.2006.07.029 
37. Domairry, G. and N. Nadim, 2007. Assessment of homotopy analysis method and homotopy perturbation method in non-linear heat transfer equation. Int. Commun. Heat Mass Transfer, 35: 93-102. DOI: $10.1016 /$ j. icheatmasstransfer.2007.06.007

38. Domairry, G. M. Ahangari and M. Jamshidi, 2007. Exact and analytical solution for nonlinear dispersive $\mathrm{K}(\mathrm{m}, \mathrm{p})$ equations using homotopy perturbation method. Phys. Lett., A, 368: 266-270. DOI: 10.1016/j.physleta.2007.04.008

39. He, J.H., 1999. Variational iteration method -a kind of non-linear analytical technique: Some examples. Int. J. Non-Linear Mech., 34: 699-708. DOI: 10.1016/S0020-7462(98)00048-1

40. He, J.H., 2000. Variational iteration method for autonomous ordinary differential systems. Applied Math. Comput., 114: 115-123. http://portal.acm.org/citation.cfm?id=353838

41. He, J.H., 1997. A new approach to nonlinear partial differential equations. Comm. Non-Linear Sci. Num. Simul., 2: 230. DOI: 10.1016/S10075704(97)90007-1

42. Dehghan, M., 2002. Fourth-order techniques for identifying a control parameter in the parabolic equations. Int. J. Eng. Sci., 40: 433-447. DOI: 10.1016/S0020-7225(01)00066-0
43. Dehghan, M., 2003. Determination of a control function in three-dimensional parabolic equations. Math. Comput. Simul., 61: 89-100. DOI: 10.1016/S0378-4754(01)00434-7

44. Lan $\mathrm{Xu}, 2007$. Application of He's parameterexpansion method to an oscillation of a mass attached to a stretched elastic wire. Phy. Lett., A., 368: 259-262. DOI: 10.1016/j.physleta.2007.04.004

45. Lan $\mathrm{Xu}, 2007$. He's parameter-expanding methods for strongly nonlinear oscillators. J. Comput. Applied Math., 207: 148-154. DOI: 10.1016/j. cam.2006.07.020

46. Lan Xu, J.H., He and A.M. Wazwaz, 2007. Variational iteration method-Reality, potential, and challenges. J. Comput. Applied Math., 207: 12. DOI: 10.1016/j.cam.2006.07.021

47. Lan $\mathrm{Xu}, 2007$. Variational iteration method for solving integral equations. Comput. Math. Applied, 54: 1071-1078.

DOI: 